\title{
Electron and phonon interactions and transport in the ultrahigh-temperature ceramic $\mathrm{ZrC}$
}

Article

Accepted Version

Mellan, T. A., Aziz, A., Xia, Y., Grau-Crespo, R. and Duff, A. I. (2019) Electron and phonon interactions and transport in the ultrahigh-temperature ceramic ZrC. Physical Review B, 99. 094310. ISSN 2469-4489 doi:

https://doi.org/10.1103/PhysRevB.99.094310 Available at https://centaur.reading.ac.uk/83012/

It is advisable to refer to the publisher's version if you intend to cite from the work. See Guidance on citing.

To link to this article DOI: http://dx.doi.org/10.1103/PhysRevB.99.094310

Publisher: APS Physics

All outputs in CentAUR are protected by Intellectual Property Rights law, including copyright law. Copyright and IPR is retained by the creators or other copyright holders. Terms and conditions for use of this material are defined in the End User Agreement.

www.reading.ac.uk/centaur 
Central Archive at the University of Reading

Reading's research outputs online 


\title{
Electron and phonon interactions and transport in ultra-high-temperature ceramic $\mathrm{ZrC}$
}

\author{
Thomas A. Mellan, ${ }^{1, *}$ Alex Aziz, ${ }^{2}$ Yi Xia, ${ }^{3}$ Ricardo Grau-Crespo, ${ }^{2}$ and Andrew I. Duff ${ }^{4}$ \\ ${ }^{1}$ Thomas Young Centre for the Theory and Simulation of Materials, \\ Department of Materials, Imperial College London, Exhibition Road, London SW7 2AZ, UK \\ ${ }^{2}$ Department of Chemistry, University of Reading, \\ Whiteknights, Reading RG6 6AD, United Kingdom \\ ${ }^{3}$ Department of Materials Science and Engineering, \\ Northwestern University, Evanston, IL 60208, USA \\ ${ }^{4}$ Scientific Computing Department, STFC Daresbury Laboratory, Hartree Centre, Warrington, UK
}

(Dated: March 12, 2019)

\begin{abstract}
We have simulated the ultra-high-temperature ceramic zirconium carbide $(\mathrm{ZrC})$ in order to predict electron and phonon scattering properties, including lifetimes and transport. Our predictions of heat and charge conductivity, which extend to $3000 \mathrm{~K}$, are relevant to extreme temperature applications of $\mathrm{ZrC}$. Mechanisms are identified on a first principles basis that considerably enhance or suppress heat transport at high temperature, including strain, anharmonic phonon renormalization and fourphonon scattering. The extent to which boundary confinement and isotope scattering effects lower thermal conductivity is predicted.
\end{abstract}

Keywords: zirconium carbide, transport, anharmonicity, phonon-phonon, electron-phonon, ultra-high temperature

\section{INTRODUCTION}

Zirconium carbide $(\mathrm{ZrC})$ is a stiff material $(E \approx 0.5$ GPa $),{ }^{1,2}$ with moderate volumetric mass density $(\rho=$ $\left.6.73 \mathrm{~g} / \mathrm{cm}^{3}\right),{ }^{3}$ metallic conductivity, ${ }^{4}$ ultra-high meltingpoint $\left(T_{m} \approx 3700 \mathrm{~K}\right),{ }^{5,6}$ and a low neutron scattering cross-section. ${ }^{7}$ Consequently, $\mathrm{ZrC}$ is relevant to the development of advanced nuclear fuel clads and aerospace materials. In each instance good thermal transport properties are essential. Hypersonic flight for example requires sharp leading edges capable of withstanding extremetemperature ablative environments, ${ }^{8,9}$ and in order to mitigate thermal shock, the material must be able to effectively transport heat from the leading edge. Similarly in the case of nuclear fuels, the clad must be able to deport thermal energy effectively at high temperatures for safe reactor operation.

High-temperature conductivity measurements are nontrivial to make so it is unsurprising that considerable scatter exists across the reported thermal data. ${ }^{4,10,11}$ Our computational predictions are therefore expected to be of practical use, as well as providing valuable theoretical insight into the basic competing factors that determine high-temperature conductivity. Advances in the computational treatment of phonons applied to thermal conductivity, ${ }^{12-14}$ provide us with a timely opportunity to present transport predictions for the prototypical ultra-high-temperature ceramic $\mathrm{ZrC}$.

In this paper we report DFT calculations on the electron and phonon scattering of phonons in bulk $\mathrm{ZrC}$. The results are used to predict charge and heat transport, elucidating grain-size and isotopic effects, as well as establishing the importance of accounting for strain, isobaric heat enhancement, and strong high-temperature anharmonicity.
This paper is set out as follows. The theoretical background and technical calculation details are listed in Sec. II $\mathrm{A}$ and Sec. IIB. Results are presented in two parts: phonon-phonon interactions and electron-phonon interactions in Sec. III A, and heat and charge transport in Sec. III B. Conclusions are presented in Sec. IV, and additional scattering information and methodological comparisons are given in the Appendix.

\section{METHODS}

\section{A. Theoretical background}

The lattice contribution to conductivity is calculated within the single-mode relaxation time approximation $^{12,15}$

$$
\kappa_{\mathrm{ph}}\left(V_{0}, T\right)=\frac{1}{N V_{0}} \sum_{\mu} C_{\mu} \mathbf{v}_{\mu} \otimes \mathbf{v}_{\mu} \tau_{\mu}^{\mathrm{ph}-\mathrm{ph}},
$$

where $\tau_{\mu}^{\text {ph-ph }}$ is the relaxation time, $\mathbf{v}_{\mu}=\hbar \partial_{\mathbf{q}} \omega_{\mu}$ is the phonon velocity and $C_{\mu}=\hbar \omega_{\mu} \partial_{T} n_{\mu}$ the heat capacity of band $\mu=n \mathbf{q}$, and $V_{0}$ is the $T=0 \mathrm{~K}$ volume of the cell at equilibrium strain.

The strain dependence of $\kappa_{\mathrm{ph}}$ is usually neglected, but in materials with large Grüneisen parameters or unusually high melting points, thermal expansion can substantially affect heat transport. In $\mathrm{ZrC}$ we account for the coupling to homogenous isotropic principle axis strains by computing $\kappa_{\mathrm{ph}}\left(V_{i}, T\right)$ at a series of volumes spanning $\left[V_{0}, V_{T_{m}}\right]$. The volume-dependent conductivity $\kappa_{\mathrm{ph}}(V, T)=\frac{1}{N V} \sum_{\mu} C_{\mathrm{V} \mu}(V, T) \mathbf{v}_{\mu} \otimes \mathbf{v}_{\mu}(V) \tau_{\mu}^{\mathrm{ph}-\mathrm{ph}}(V, T)$ is calculated by a simple procedure that linearly mixes 


$$
\kappa_{\mathrm{ph}}(V, T)=\sum_{i}\left\{\kappa_{\mathrm{ph}}\left(V_{i}, T\right)\left(1-t_{i, i+1}(T)\right)+\kappa_{\mathrm{ph}}\left(V_{i+1}, T\right) t_{i, i+1}(T)\right\} \operatorname{box}(T) .
$$

$t_{i, i+1}(T)$ is a mixing parameter, $t_{i, i+1}(T)=\frac{T-T_{i}}{T_{i+1}-T_{i}}$, that interpolates the temperature dependence of $\kappa_{\mathrm{ph}}$ between the pairs of volumes (temperatures). The box function selects the interpolation temperature range $\left[T_{i}, T_{i+1}\right]$ as box $(T)=\Theta\left(T-T_{i}\right)-\Theta\left(T-T_{i+1}\right)$.

As well as the effect of volume expansion on phonon thermal conductivity, strong anharmonic effects become increasingly important at high temperature. We account for phonon frequency renormalization at finite temperature by explicitly considering anharmonicity up to fourth order using a recently developed real-space-based anharmonic phonon renormalization scheme. ${ }^{16,17}$ The required high-order anharmonic interatomic force constants were constructed using compressive sensing lattice dynamics (CSLD). ${ }^{18}$ Due to the computational expense, we only performed calculations at selected temperatures, for example, in the low $(300 \mathrm{~K})$, medium $(1500 \mathrm{~K})$, and high $\left(T_{m}\right)$ temperature regimes. Moreover, we explicitly calculated the intrinsic phonon scattering rates from fourphonon processes, as recently formulated by Feng and Ruan, ${ }^{19}$ beyond the regularly used three-phonon scatterings. To estimate the impacts of anharmonic renormalization and four-phonon scattering on conductivity at a range of temperatures, the effects on $\kappa_{\mathrm{ph}}(T)$ are interpolated in temperature between the weakly and strongly anharmonic regimes, analogous to the interpolation specified in Eqn. 2. by

The isobaric phonon conductivity $\kappa_{\mathrm{ph}}(p)$ is calculated

$$
\kappa_{\mathrm{ph}}(p)=\gamma \kappa(V)
$$

The enhancement factor, $\gamma=C_{\mathrm{P}} / C_{\mathrm{V}}$, is determined using $C_{\mathrm{P}}$ computed at the quasi-harmonic level of theory.

Three-phonon relaxation lifetimes $\left(\tau_{\mu}^{\text {ph-ph }}=\right.$ $1 / 2 \Gamma^{\prime \prime}$,ph-ph) are calculated based on the imaginary self-energies, $\Gamma^{\prime \prime}$,ph-ph $\equiv \operatorname{Im} \Gamma^{\text {ph-ph }} \cdot{ }^{12,15} \Gamma^{\prime \prime}$,ph-ph is computed both by strain-dependent third-order lattice dynamics, ${ }^{12,15}$ and by CSLD to also account for hightemperature anharmonicity. ${ }^{18}$ Four-phonon scattering times are computed by iterative solution to the BTE, ${ }^{16}$ at selected temperatures only $(300 \mathrm{~K}, 1500 \mathrm{~K}$ and $3800 \mathrm{~K})$. Three and four-phonon scattering times are combined using Matheisen's rule, and the total phonon relaxation lifetime including other terms is estimated as

$$
\tau_{\mu}=\frac{1}{\sum_{i} 1 / \tau_{\mu}^{i}}
$$

for $i \in\{$ ph-ph, ph-iso, ph-boundary\}. Isotope mass defect scattering is treated perturbatively, ${ }^{12,20}$ and boundary scattering as if providing a restriction on $\tau_{\mu}^{\text {ph-boundary }}=L / \mathbf{v}_{\mu}$ by domain size $L$.

The total thermal conductivity $\kappa_{\text {total }}$ is computed as

$$
\kappa_{\text {total }}=\kappa_{\mathrm{ph}}+\kappa_{\mathrm{el}},
$$

with electron thermal conductivity $\kappa_{\mathrm{el}}$ treated semiclassically $^{21,22}$

$$
\kappa_{\mathrm{el}}=\frac{1}{V T} \int d \varepsilon(\varepsilon-\mu)^{2}\left(-f^{\prime}\right) \sum_{\lambda} \mathbf{v}_{\lambda} \otimes \mathbf{v}_{\lambda} \tau_{\lambda} \delta\left(\varepsilon-\varepsilon_{\lambda}\right)
$$

and electrical conductivity is computed similarly ${ }^{21,22}$

$$
\sigma_{\mathrm{el}}=\frac{1}{V} \int d \varepsilon\left(-f^{\prime}\right) \sum_{\lambda} \mathbf{v}_{\lambda} \otimes \mathbf{v}_{\lambda} \tau_{\lambda} \delta\left(\varepsilon-\varepsilon_{\lambda}\right),
$$

where $\lambda$ subsumes wavevector and band quantum numbers $\lambda \equiv\{n, \mathbf{k}\}, f^{\prime}=\partial_{\varepsilon} f(T, \varepsilon)$ is the electron occupancy energy derivative, $\mathbf{v}_{\lambda}=\partial_{\mathbf{k}} \varepsilon_{n \mathbf{k}}$ is the band velocity tensor, and $\tau_{\lambda}$ is the electron relaxation time. The effect of thermal expansion on $\kappa_{\mathrm{el}}$ and $\sigma_{\mathrm{el}}$ is accounted for by computing each quantity for a series of volumes along the quasiharmonic thermal expansion curve. Conductivity tensors are determined, up to a factor of $\tau_{\lambda}$, from local density approximation (LDA) band structures using Wannier functions, with methodological comparison to Bloch functions and DFPT in Appendix. ${ }^{22-27}$ The relaxation time $\tau_{\lambda}$ is equated with the electronphonon scattering time $\tau_{\lambda}^{\mathrm{el}-\mathrm{ph}} . \tau_{\lambda}^{\mathrm{el}-\mathrm{ph}}$ is determined from $\tau_{\lambda}^{\text {el-ph }}=1 / 2 \Sigma_{\lambda}{ }^{\prime \prime}$,el-ph , with the imaginary part of the selfenergy, $\Sigma_{\lambda}^{\prime \prime}$,el-ph $\equiv \operatorname{Im} \Sigma_{\lambda}^{\text {el-ph }}$, found using the method of Poncé et al. ${ }^{28,29}$. This method is also used to compute $\Pi_{\mu}^{\prime \prime}$,el-ph, to determine the phonon relaxation time from electron-phonon scattering.

\section{B. Technical details}

Three-phonon scattering rates are computed using the second-order perturbation theory implemented by the PHONO3PY code. ${ }^{12,15}$ Small-displacement third-order force constants are calculated at seven volumes that span the range of thermal expansion. For third-order force constants at each dilation, 144 displacements are made on the $2 \times 2 \times 2$ (64 atom) of $\mathrm{ZrC}$ supercell. Second-order force constant displacements are made on a $4 \times 4 \times 4(512$ atom) supercell at each volume. q-points are sampled at a density equivalent to a $31 \times 31 \times 31$ grid for the conventional eight atom unit cell. 
Compressive sensing lattice dynamics (CSLD) ${ }^{18}$ is used to account for strong anharmonic effects at high temperature, with force constant tensors (FCTs) up to sixth order determined from snap-shots of uncorrelated quasi-random configurations. Convergence is achieved for $\mathrm{ZrC}$ by twenty configurations of a 128 atom supercell $(4 \times 4 \times 4$ of the primitive cell $)$

Force-constants are calculated using the VASP density functional theory (DFT) code. ${ }^{25,26}$ The PZ81 LDA ${ }^{30}$ functional provides a satisfactory description of $\mathrm{ZrC}$ at low temperature, giving a zero-point-corrected $T=0 \mathrm{~K}$ value of $a_{\mathrm{LDA}}=4.667 \AA$, compared to an experimentally reported value of $a_{\exp }=4.694 \AA{ }^{6}$ The difference is similar in magnitude but opposite in sign to $\mathrm{PBE},{ }^{31}$ however we choose to work with the LDA due to reported superior description of thermodynamics at high temperatures. ${ }^{32}$

DFT calculations employ the projector-augmented wave (PAW) method, ${ }^{33}$ with $4 s$ and $4 p$-Zr electron included as valence states. Kinetic energy is cutoff above $700 \mathrm{eV}$ and k-points are sampled at a density commensurate to a $12 \times 12 \times 12$ mesh for the conventional cell. Methfessel-Paxton smearing is applied with $0.2 \mathrm{eV}$ broadening. ${ }^{34}$ Cell total energies and individual eigenvalues are converged to $10^{-8} \mathrm{eV}$, and force differences to $10^{-6} \mathrm{eV} / \AA$

Electron-phonon lifetimes are calculated using the Electron-Phonon-Wannier (EPW) code, interfaced with Quantum-Espresso ${ }^{28}$ (QE). ${ }^{29} \mathbf{k}$-space interpolation uses maximally-localized Wannier functions generated using WANNIER90. ${ }^{23,24,35-37}$

QE calculations use an LDA exchange-correlation functional, ${ }^{30,38}$ with a projected augmented wave (PAW) pseudopotential for $\mathrm{Zr}$ with $4 s^{2} 4 p^{6} 4 d^{2} 5 s^{2}$ electrons considered as valence electrons, and a norm-conserving pseudopotential for $\mathrm{C}$ used with $2 s^{2} 2 p^{2}$ electrons treated as valence electrons. Convergence of $0.5 \mathrm{mRy}$ is obtained with a $12 \times 12 \times 12 \Gamma$-centered $\mathbf{k}$-point mesh and a kinetic energy cutoff of $200 \mathrm{Ry}$. Ionic minimization is performed until energy differences are less than $10^{-6} \mathrm{Ha}$ and force differences less than $10^{-5} \mathrm{Ha} /$ Bohr. Electronic convergence is at least $10^{-10} \mathrm{Ry}$.

Electron-phonon interaction strengths are found using dynamical matrices from DFPT. ${ }^{39-41}$ An irreducible $\Gamma$ centered $6 \times 6 \times 6$ q-point mesh is used with convergence criteria of at least $10^{-14} \mathrm{Ry}$. Non-self-consistent calculations are performed on a coarse $\Gamma$-centered $6 \times 6 \times 6$ k-point mesh using the same energy criteria as the energy minimization. Both the coarse $\mathbf{q}$-point and $\mathbf{k}$-point meshes were tested for convergence. These results are then used for Wannier interpolation.

The Wannier functions are projected onto carbon $s p^{3}$ orbitals and three $\mathrm{Zr} d$-orbitals. Four bands below the Fermi level representing the $\mathrm{Zr} s$-band and the three $\mathrm{Zr}$ $p$-bands are not included in the calculation as well as the highest conduction band. In each calculation the disentangled method is used ${ }^{37}$ and the disentangled energy window is set to between $12 \mathrm{eV}$ below the Fermi level to include the $\mathrm{C} s$-orbital and $2 \mathrm{eV}$ above the Fermi level.
These settings provide the best spreads (between 1.5-1.7 $\AA^{2}$ per Wannier function). As Wannier interpolation on homogeneous $\mathbf{k}$ and $\mathbf{q}$-grids of $45 \times 45 \times 45$ were unable to achieve convergence, randomly generated grids were used as suggested by Poncé et al.. ${ }^{28}$ A grid of 50,000 randomly generated $\mathbf{k}$-points and 150, 000 randomly generated q-points with a broadening of $20 \mathrm{meV}$ is sufficient for convergence.

The conductivity tensor in Eq. 7 is determined from VASP-calculated ${ }^{25,26}$ LDA $^{30}$ band structures. The technical parameters are identical to the VASP phonon calculation details described previously except for the reciprocal-space sampling density. The electronic band structure calculations employed a dense k-point mesh sampling of $39 \times 39 \times 39$, from which the conductivity tensor is determined by i) linear response routines in VASP ${ }^{27}$ ii) reciprocal-space band velocities via BoltzTrAP, ${ }^{21}$ and iii) by real-space band velocities via BoltzWAnN. ${ }^{22-24}$ Electrical transport tensors account for the thermal expansion of the lattice although the effect is marginal.

\section{RESULTS}

\section{A. Phonon-phonon and electron-phonon scattering}

The acoustic-type phonon bands in $\mathrm{ZrC}$, which project more than $98 \%$ onto the motion of $\mathrm{Zr}$ atoms, are weakly interacting compared to the carbon-projecting optic-type phonon dispersion bands. This is shown for $\mathrm{ZrC}$ at $10 \mathrm{~K}$ and at $300 \mathrm{~K}$ by the phonon-phonon interaction broadened dispersion in Fig. 1a-b. Optic-type modes at $\mathbf{q}=0$ are the most strongly interacting for $T>300 \mathrm{~K}$. For $T<300 \mathrm{~K}$, other lower symmetry wavevectors, for instance between $K-\Gamma$ and $L-\Gamma$ in the Brillouin zone, become more actively scattering than the zone-center $\Gamma$ point optic modes in Fig. 1a-b.

The linear temperature dependence of the $\mathrm{ZrC}$ linewidths is shown for a range of wavevectors in Fig. 1c, with average values comparable to those reported for other materials. ${ }^{12}$ For example the average three-phonon linewidth is $0.05 \mathrm{THz}$ at $300 \mathrm{~K}$, which is lower than the $\mathrm{CuCl} 300 \mathrm{~K}$ linewidth $(1.3 \mathrm{THz})$ and slightly larger than GaAs $(0.03 \mathrm{THz})$. In $\mathrm{ZrC}$ we emphasize the limited insight from quoting $n \mathbf{q}$ averaged values, as the linewidths are quite strongly anisotropic (as pictured, Fig. 1b). On the validity of the perturbative quasi-particle formalism within which lifetimes are determined, acoustic modes are weakly interacting to $T_{m}$ but caution is necessary on the interpretation of optic modes at high-symmetry points for which linewidths can be of the order $1 \mathrm{THz}$ for temperatures exceeding $0.6 T_{\mathrm{m}}$.

$\mathrm{ZrC}$ is unusual in exhibiting ceramic and metallic bonding characteristics. To describe the electrical conductivity of $\mathrm{ZrC}$ we account for electron-phonon scattering. The average time for the scattering of electrons by 

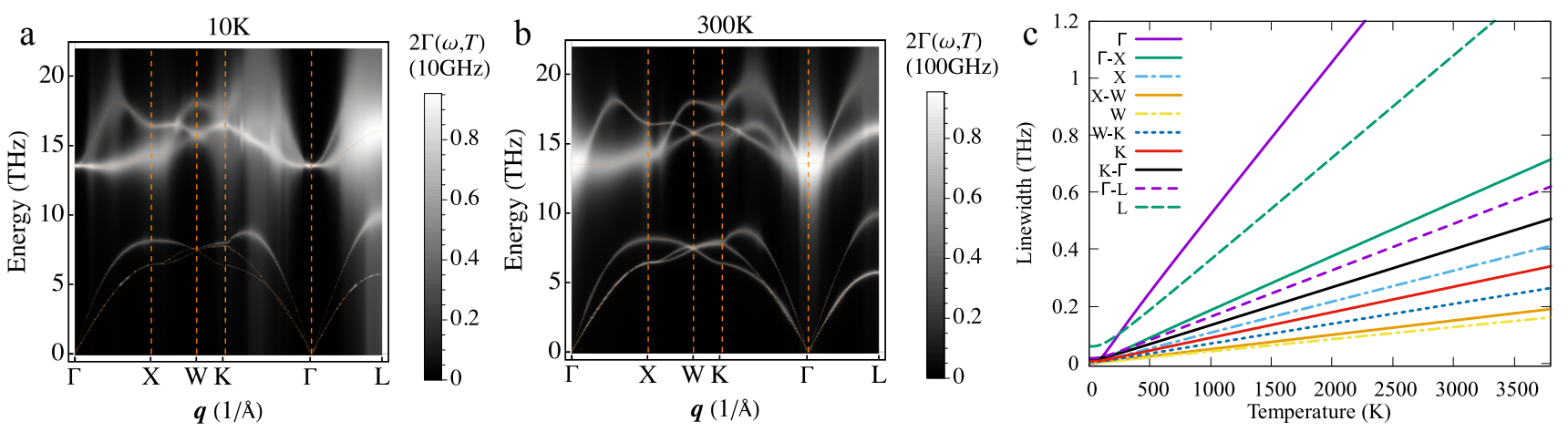

Figure 1. a-b) Interaction broadened harmonic phonon dispersion at $10 \mathrm{~K}$ and $300 \mathrm{~K}$. FWHM $\Gamma_{\lambda}^{\prime \prime}$,ph-ph $(\omega, \mathbf{q}, T)$ line broadening is Lorentzian and specified by the contour brightness scale on the right axis which ranges from $0-10 \mathrm{GHz}$ for $10 \mathrm{~K}$ to $0-100 \mathrm{GHz}$ for $300 \mathrm{~K}$. c) Linewidth $v s$ temperature at selected points (labelled) in the Brillouin zone.

phonons, calculated by

$$
\tau^{\mathrm{el-ph}}(T)=\int d \varepsilon \frac{1}{N_{n \mathbf{k}}} \sum_{n, \mathbf{k}} \tau_{n \mathbf{k}}^{\mathrm{el-ph}}(T) \delta\left(\varepsilon-\varepsilon_{n, \mathbf{k}}\right) f^{\prime}(\varepsilon, T)
$$

is shown in Fig. 2a. The average relaxation time decreases with temperature, for example from $\tau^{\mathrm{el}-\mathrm{ph}}(500 \mathrm{~K})=6.3$ fs to $\tau^{\mathrm{el}-\mathrm{ph}}(2500 \mathrm{~K})=1 \mathrm{fs}$. As $\mathrm{ZrC}$ is semi-metallic, $\tau^{\text {el-ph }}(T)$ is enhanced with temperature as $f^{\prime}=\partial_{\varepsilon} f(T, \varepsilon)$ samples states that increase in concentration away from the Fermi energy. This is demonstrated in Fig. 2a-inset in the electron self-energy $\Sigma^{\prime \prime}$,el-ph which is a local minimum about the Fermi energy. Recent work has shown intrinsic defects such as Frenkel defects, which are predicted to spontaneously generate at high temperature in $\mathrm{ZrC}$, can as much double the density of states at the Fermi energy. ${ }^{42}$ This is likely to modulate $\Sigma^{\prime \prime}$,el-ph and electron transport at high temperature, though explicit characterisation with first principles calculations is beyond the scope of this work.

In experiments the width of a phonon line may be measurable, while the origin of the broadening remains obscured, so it is interesting to compare phonon linewidths from electron-phonon interactions $2 \Pi^{\prime \prime}$,el-ph and from three-phonon interactions $2 \Gamma^{\prime \prime}$,ph-ph. Values for $2 \Gamma^{\prime \prime}$, ph-ph and $2 \Pi^{\prime \prime}$,el-ph are listed for selected temperatures in Table I. At $300 \mathrm{~K}, 2 \Gamma_{\mathbf{q}=\mathbf{0} \text {,optic }}^{\prime \prime}$ peak values are smaller $($ ca. $\times 3)$ than $2 \Pi_{\mathbf{q}=\mathbf{0} \text {,optic }}^{\prime \prime}$, but for higher temperatures phononphonon interaction is many times greater than electronphonon (ca. $\times 8)$. As a crystal with partially occupied states at the Fermi energy, it is a point of interest that the room temperature total phonon linewidth has comparable contributions from anharmonic and electron-phonon interactions. This observation for the linewidth in conducting crystalline systems is however not exceptional, and has recently been reported at room temperature in a number of systems, including graphite, ${ }^{43,44}$ noble metals with small $\operatorname{DOS}\left(E_{\mathrm{F}}\right)$ such as $\mathrm{Cu}, \mathrm{Ag}$ and $\mathrm{Au},{ }^{45-47}$ as well as more exotic systems such as the superconductor palladium hydride. ${ }^{43}$
Table I. Phonon linewidths from electron-phonon and phonon-phonon interactions.

\begin{tabular}{ccc}
\hline$T$ & $2 \Pi_{\mathbf{q}=0, \text { optic }}^{\prime \prime}$, el-ph & $2 \Gamma_{\mathbf{q}=0, \text { optic }}^{\prime \prime}$, ph-ph \\
\hline \hline $300 \mathrm{~K}$ & $0.50 \mathrm{THz}\left(17 \mathrm{~cm}^{-1}\right)$ & $0.17 \mathrm{THz}\left(5.7 \mathrm{~cm}^{-1}\right)$ \\
$3000 \mathrm{~K}$ & $0.32 \mathrm{THz}\left(11 \mathrm{~cm}^{-1}\right)$ & $2.4 \mathrm{THz}\left(80 \mathrm{~cm}^{-1}\right)$ \\
\hline
\end{tabular}

\section{B. Heat and charge transport}

Unlike most ceramics $\mathrm{ZrC}$ is a relatively good conductor of electricity. ${ }^{4,6,10,48}$ We have calculated the electrical conductivity $\left(\sigma_{\mathrm{el}}\right)$ of $\mathrm{ZrC}$ within a first principles Boltzmann transport approach, using the Wannier function DFT method to determine the electron-phonon relaxation time and band velocities. This method is compared to other approaches in the Appendix. The predicted values of $\sigma_{\mathrm{el}}$ range from $\sigma_{\mathrm{el}}(500 \mathrm{~K})=1.4 \times 10^{-6} \Omega^{-1} \mathrm{~m}^{-1}$ to $\sigma_{\text {el }}(2500 \mathrm{~K})=3.8 \times 10^{-7} \Omega^{-1} \mathrm{~m}^{-1}$, with $\sigma_{\text {el }}(T)$ shown in Fig. 3a, alongside experimentally measured values from multiple sources. ${ }^{4,6,10,48}$ The electron thermal conductivity $\kappa_{\mathrm{el}}(T)$ is shown in Fig. 3b. Starting at zero temperature $\kappa_{\mathrm{el}}(T)$ exhibits a sharp increase initially, then increases almost linearly, rising from $\kappa_{\mathrm{el}}(500 \mathrm{~K})=$ $17 \mathrm{Wm}^{-1} \mathrm{~K}^{-1}$ to $\kappa_{\mathrm{el}}(2500 \mathrm{~K})=40 \mathrm{Wm}^{-1} \mathrm{~K}^{-1}$.

In each instance the $\sigma_{\mathrm{el}}$ and $\kappa_{\mathrm{el}}$ values in Fig. 3ab computed from first principles give reasonable agreement with experiment. This even appears to be true as the system nears the Mott-Ioffe-Regel limit where resistivity usually saturates. ${ }^{48-52}$ The physical origin of additional transport channels leading to saturation can be somewhat debatable but is often well-described in an empirical sense by a parallel shunt model. It is therefore likely our high-temperature conductivity predictions are a lower limit for the conductivity of the defect-free crystal. The reasonable agreement of our transport predictions with experimentally reported ones at high temperatures is partially explained by electron quasi-momentum quantum numbers that remain moderately good up to 

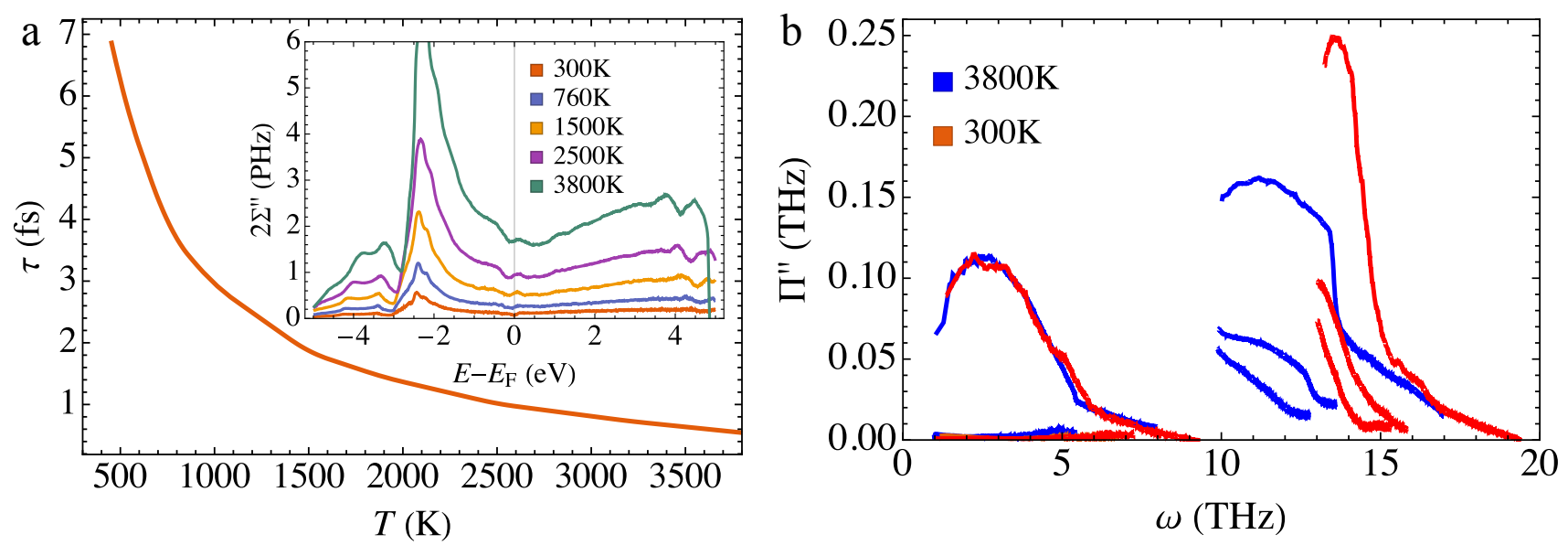

Figure 2. a) Electron relaxation time and associated imaginary self-energy $\Sigma_{\lambda}^{\prime \prime}$,el-ph $(\varepsilon, T)$ inset. b) Imaginary phonon self-energy $\Pi^{\prime \prime}$, el-ph $(\omega, T)$, for each of the three acoustic and optic bands.
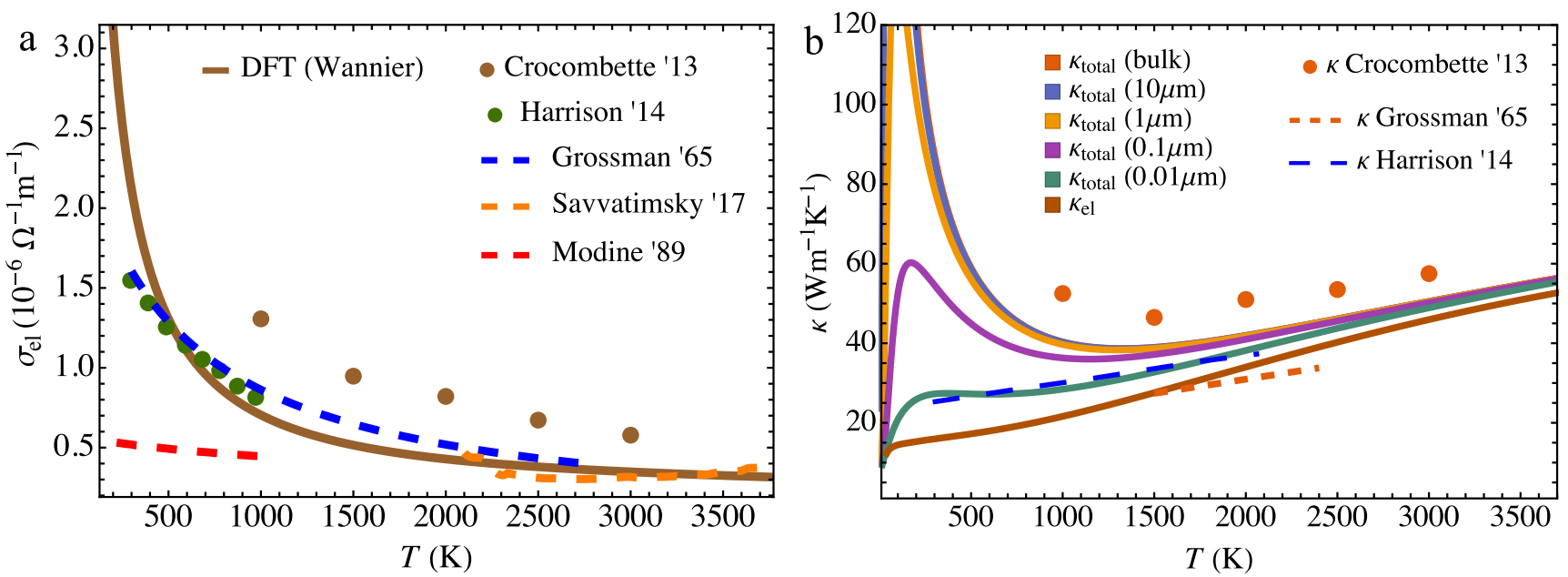

Figure 3. a) Electrical conductivity $\sigma_{\mathrm{el}}$ predictions (solid line). b) Thermal conductivity predictions for $\kappa_{\mathrm{total}}=\kappa_{\mathrm{ph}}+\kappa_{\mathrm{el}}$ and $\kappa_{\mathrm{el}}$. Dashed and dotted lines denote the experimental measurements ${ }^{4,6,10,48}$ and computed results of Crocombette. ${ }^{11}$

quite high temperatures - for example, at $3000 \mathrm{~K}$ the electron mean-free path is still ca. $\times 3 a$ lattice parameters. Above this temperature the electron transport plots in Fig. 3a-b are extended for reference only and with underlined caution due to the inadequacy of the Boltzmann electron transport picture close to $T_{m}$ when scattering lengths and lattice parameters become close.

The total phonon and electron thermal conductivity, $\kappa_{\text {total }}=\kappa_{\mathrm{ph}}+\kappa_{\mathrm{el}}$, is shown versus temperature in Fig. 3b. Whether $\kappa_{\mathrm{ph}}$ or $\kappa_{\mathrm{el}}$ is the larger contribution to $\kappa_{\text {total }}$ depends on temperature and other factors such as geometric constraints. For example, grain boundaries introduce a restriction on maximum phonon path length for weakly scattering $\mathrm{Zr}$ modes that considerably changes $\kappa_{\mathrm{ph}}$. Large domains $(L \geqq 10 \mu \mathrm{m})$ permit the transport of heat by long-wavelength highvelocity modes in $\mathrm{ZrC}$, resulting in high values such as $\kappa_{\text {total }}(300 \mathrm{~K})=87 \mathrm{Wm}^{-1} \mathrm{~K}^{-1}$. Grains of moderate sizes suppress phonon transport at low temperatures, with our model predicting $\kappa_{\text {total }}(300 \mathrm{~K})=55 \mathrm{Wm}^{-1} \mathrm{~K}^{-1}$ at $L=0.1 \mu \mathrm{m}$. Small domain sizes such as $L=0.01 \mu \mathrm{m}$ severely limit transport processes from weakly scattering high-velocity modes, further lowering $\kappa_{\text {total }}(300 \mathrm{~K})$ to $27 \mathrm{Wm}^{-1} \mathrm{~K}^{-1}$. The size dependence of $\kappa_{\text {total }}$ suggests grain control by sintering or synthesis temperature is important to design the transport behavior of $\mathrm{ZrC}$. More details on grain boundary scattering and the extent of mean-free path saturation expected at high temperature are given in the Appendix.

In Fig. 4 we show a range of temperature-dependent mechanisms that can enhance or suppress phonon thermal conductivity. Note, each effect that is analyzed has been included the prior calculation of $\kappa_{\text {total }}$ that was presented Fig. 3b.

Dilation of $\mathrm{ZrC}$ by tensile principal axis strains is found to lower $\kappa_{\text {ph }}$ considerably. For instance thermal expan- 
sion decreases phonon conductivity by up to $65 \%$ relative to $\kappa_{\mathrm{ph}}$ at the $0-\mathrm{K}$ equilibrium volume, as shown by the enhancement factor $\kappa_{\mathrm{qha}}^{\mathrm{ph}}[V(T)] / \kappa^{\mathrm{ph}}\left(V_{0}\right)$ versus temperature in Fig. 4a. One way to rationalize the large change is by considering how the stiffness (specifically, isothermal bulk modulus $K_{\mathrm{T}}$ ) and volumetric mass density $(\rho)$ change with temperature relative to each other. Provided $\left|\frac{\partial K_{\mathrm{T}}}{\partial T}\right| /\left|\frac{\partial \rho}{\partial T}\right|<1, \kappa_{\mathrm{ph}}$ decreases with thermal expansion. Indeed this is the case and a decrease of 39 $\%$ is observed from $T=10 \mathrm{~K}$ to $T_{m}$. As $K_{\mathrm{T}} / \rho$ is equal to the long wavelength-limit band velocity $v_{s}^{2}$, the decrease in $\sqrt{K_{\mathrm{T}} / \rho}$ is equivalent to decrease in acoustic band velocity from $52.1 \mathrm{THz} \AA(5210 \mathrm{~m} / \mathrm{s})$ to $44.1 \mathrm{THz} \AA(4410$ $\mathrm{m} / \mathrm{s}$ ). This drop accounts for a considerable part of the decrease in $\kappa_{\text {ph }}$ with temperature in Fig. 4 a.

Quantum zero-point motion modifies the equilibrium configuration through tensile strain. In $\mathrm{ZrC}$ this softens modes and decreases $\kappa_{\text {ph }}$ by a temperature-independent factor of ca. 5\%, which is shown in Fig. 4b. Conversely the isobaric heat capacity enhancement factor (see Eqn. 3 ) is negligible at low temperature, but increases $\kappa_{\mathrm{ph}}$ by as much as $35 \%$ at high temperature, as shown in Fig. 4f.

At low temperature mass scattering at the natural isotopic abundance in $\mathrm{ZrC}$ is important. For instance $\kappa_{\mathrm{ph}}$ in Fig. 4c is more than $50 \%$ lower than an artificially prepared isotopically pure crystal at low temperature, but effect quickly falls off with increasing temperature. Grain size effects are also substantial at low temperature, as illustrated in the prior discussion mean-free path constraints for Fig. 3b. For length scale effects from the opposite limit, minimum phonon conductivity saturation $^{53,54}$ is expected to be comparatively smaller even at high temperatures. This is based on the accumulated phonon thermal conductivity as a function of mean-free path, which is given in Fig. 9, in the Appendix.

For high temperatures $\left(>0.5 T_{\mathrm{m}}\right)$ perturbative thirdorder lattice dynamics becomes insufficient as the system explores atomic displacements far from the equilibrium configuration. Strong anharmonicity renormalizes dispersion bands to higher frequencies, opposite to the $\frac{\partial \ln |\omega|}{\partial \ln V}<0$ typical volume softening of frequencies with positive thermal expansion. The high-temperature anharmonic renormalization considerably enhances $\kappa_{\mathrm{ph}}$ as shown in Fig. 4d. The effect as a fraction of the hightemperature conductivity can be large, for example increasing $\kappa_{\text {ph }}$ by ca. $100 \%$ for temperatures in excess of $2000 \mathrm{~K}$.

It has recently been noted that four-phonon scattering plays a critical role in determining the lattice thermal conductivities in both weakly and strongly anharmonic systems, e.g., $\mathrm{BAs}^{19}$ and $\mathrm{PbTe}^{16}$ respectively. In $\mathrm{ZrC}$ we observe that four-phonon scattering processes become very prominent at high temperature for $\kappa_{\mathrm{ph}}$. As with PbTe,${ }^{16}$ renormalization enhances $\kappa_{\mathrm{ph}}$ and four-phonon processes suppress $\kappa_{\mathrm{ph}}$. The degree of cancellation be-

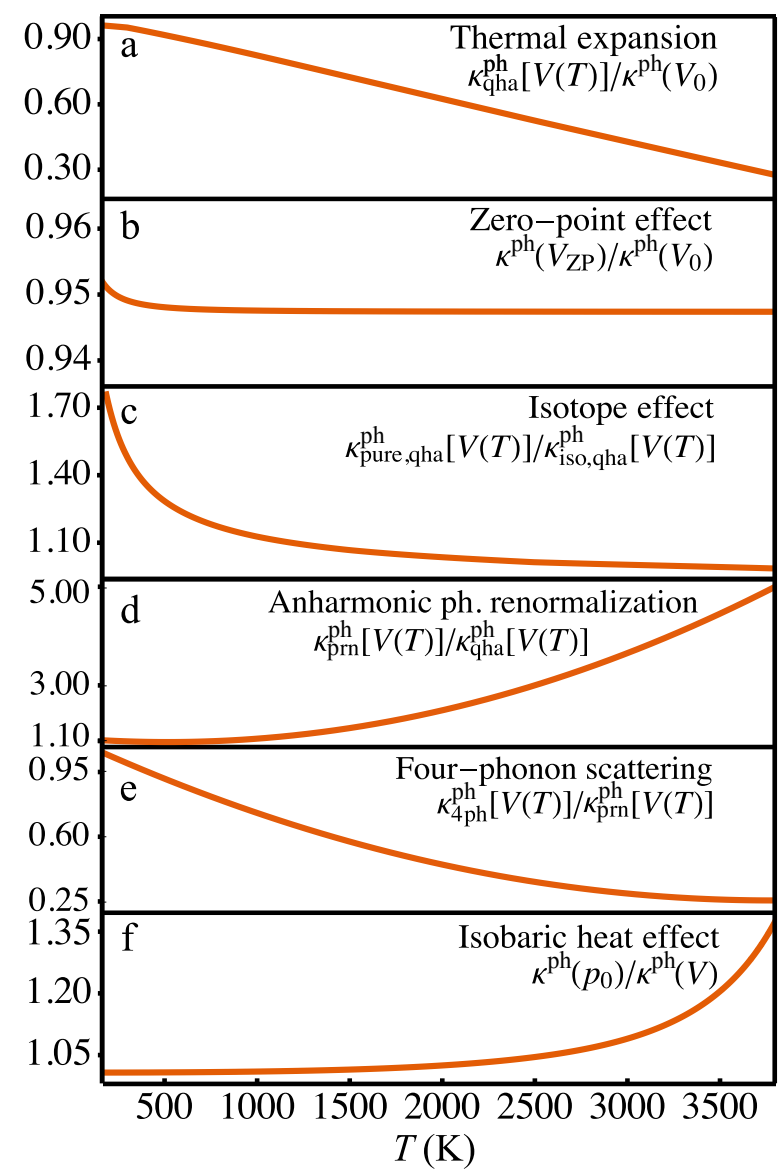

Figure 4. Phonon thermal conductivity enhancement and suppression mechanisms in ZrC. a) Thermal expansion lowers conductivity by $\kappa_{\text {qha }}^{\mathrm{ph}}[V(T)] / \kappa^{\mathrm{ph}}\left(V_{0}\right)$. b) Zeropoint motion lowers conductivity by $\kappa^{\mathrm{ph}}\left(V_{\mathrm{ZP}}\right) / \kappa^{\mathrm{ph}}\left(V_{0}\right)$. c) Isotope purity enhances conductivity by factor of $\kappa_{\text {pure,qha }}^{\mathrm{ph}}[V(T)] / \kappa_{\text {iso,qha }}^{\mathrm{ph}}[V(T)]$ compared mass scattering at the natural isotopic abundance. d) Anharmonic phonon renormalization enhances conductivity by $\kappa_{\mathrm{prn}}^{\mathrm{ph}}(V) / \kappa_{\mathrm{qha}}^{\mathrm{ph}}(V)$ relative to a crystal with quasiharmonic frequency dependence. e) Additionally including four-phonon scattering suppresses conductivity by $\kappa_{4 \mathrm{ph}}^{\mathrm{ph}}(V) / \kappa_{\mathrm{prn}}^{\mathrm{ph}}(V)$. f) Isobaric heat effect enhances conductivity $\kappa^{\mathrm{ph}}\left(p_{0}\right) / \kappa^{\mathrm{ph}}(V)$.

tween these anharmonic effects can be observed by considering Fig. 4d-e, and is illustrated by noting the renormalization enhancement of $\kappa_{\mathrm{ph}}$ at $300 \mathrm{~K}(3800 \mathrm{~K})$ is +4.2 $\mathrm{W} / \mathrm{mK}(+6.2 \mathrm{~W} / \mathrm{mK})$, while four-phonon scattering lowers $\kappa_{\text {ph }}$ by $-4.1 \mathrm{~W} / \mathrm{mK}(-5.8 \mathrm{~W} / \mathrm{mK})$. Additional details on four-phonon scattering and anharmonic phonon renormalization are given in the Appendix. 


\section{CONCLUSIONS}

We have reported first principles calculations on the scattering and transport properties of electrons and phonons in the ultra-high-temperature ceramic $\mathrm{ZrC}$. The nature of the phonon linewidth in $\mathrm{ZrC}$ has been examined in terms of the energy dependence, anisotropy across the Brillouin zone and temperature dependence of phononphonon and electron-phonon interactions. In each instance phonons primarily scatter via optic modes at the $\Gamma$ point. The total phonon linewidth is predominantly phonon-phonon in character rather than electron-phonon for all but the lowest temperatures.

The electrical and thermal conductivities $\sigma_{\mathrm{el}}$ and $\kappa_{\mathrm{el}}$ have been predicted at ambient pressure as a function of temperature, along with total thermal conductivity $\kappa_{\text {total }}=\kappa_{\mathrm{el}}+\kappa_{\mathrm{ph}}$. Thermal expansion of crystal volume notably suppresses thermal conductivity, decreasing the phonon contribution by more than $50 \%$ at $0.75 T_{\mathrm{m}}$. Suppression of thermal conductivity by strain sources, such as thermal expansion and grain boundaries, should be considered when engineering heat dissipation of an ultra-high temperature ceramic for extreme environment applications. At low temperature $\kappa_{\mathrm{ph}}$ is considerably lowered by isotope mass defect scattering, and by features that enforce geometric constraints such as grain boundaries that prevent the crystal supporting longlived phonon modes. Isobaric heat capacity, and anharmonic phonon renormalization, provide substantial enhancements in $\kappa_{\mathrm{ph}}$ at high temperature (ca. $0.75 T_{\mathrm{m}}$ ). Four-phonon scattering strongly suppresses thermal con- ductivity at high temperature, almost cancelling the anharmonic frequency renormalization effect.

The examination of point and extended defects and sub-stoichiometry on transport is beyond the scope of this work, but would be a valuable future extension to this work. As would the examination of saturation effects and non-quasiparticle transport for $T>0.75 T_{m}$.

\section{ACKNOWLEDGEMENTS}

T.A.M. acknowledges the financial support of EPSRC Programme Grant Material Systems for Extreme Environments (XMat) (Grant No. EP/K008749/2), EPSRC Programme Grant Carbides for Future Fission Environments (CAFFE) (Grant No. EP/M018563/1), and H2020 project Il Trovatore (Grant No. 740415). T.A.M. acknowledges computational resources from the UK Materials and Molecular Modelling Hub (Grant No. EP/P020194/1) and embedded CSE 33 of the ARCHER UK National Supercomputing Service (http://www.archer.ac.uk). A.I.D. acknowledges support from the STFC Hartree Centre's Innovation: Return on Research programme, funded by the UK Department for Business, Energy \& Industrial Strategy.

\section{REFERENCES}

* t.mellan@imperial.ac.uk

1 D. Cheng, S. Wang, and H. Ye, J. Alloy Compd. 377, 221 (2004).

2 D. Sciti, S. Guicciardi, and M. Nygren, Scrip. Mat. 59, 638 (2008).

${ }^{3}$ D. R. Lide and W. M. Haynes, CRC handbook of chemistry and physics (2009).

${ }^{4}$ R. W. Harrison and W. E. Lee, Adv. Appl. Cer. 115, 294 (2016).

5 A. Fernández Guillermet, J. Alloy Compd. 217, 69 (1995).

6 A. I. Savvatimskiy, S. V. Onufriev, and S. A. Muboyadzhyan, J. Mater. Res. 32, 1557 (2017).

7 C. R. F. Azevedo, Eng. Fail. Anal. 18, 1943 (2011).

8 A. Paul, D. D. Jayaseelan, S. Venugopal, E. Zapata-Solvas, J. Binner, B. Vaidhyanathan, A. Heaton, P. Brown, and W. E. Lee, Amer. Ceri. Soc. Bull. 91, 22 (2014).

9 J. F. Justin and A. Jankowiak, Aero. J. 3, 1 (2011).

10 L. N. Grossman, J. Amer. Cer. Soc. 48, 236 (1965).

11 J. Crocombette, J. Phys: Cond. Matt. 25, 505501 (2013).

12 A. Togo, L. Chaput, and I. Tanaka, Phys. Rev. B 91, 094306 (2015).

13 T. Tadano, Y. Gohda, and S. Tsuneyuki, J. Phys: Cond. Matt. 26, 225402 (2014).

14 W. Li, J. Carrete, N. A. Katcho, and N. Mingo, Comp. Phys. Comm. 185, 1747 (2014).
15 L. Chaput, A. Togo, I. Tanaka, and G. Hug, Phys. Rev. B 84, 094302 (2011).

${ }^{16}$ Y. Xia, Appl. Phys. Lett. 113, 073901 (2018).

17 Y. Xia and M. K. Y. Chan, Appl. Phys. Lett. 113, 193902 (2018).

18 F. Zhou, W. Nielson, Y. Xia, and V. Ozolins, Phys. Rev. Lett. 113, 185501 (2014).

19 T. Feng, L. Lindsay, and X. Ruan, Phys. Rev. B 96, 161201 (2017).

${ }^{20}$ M. Cardona and M. L. W. Thewalt, Revi. Mod. Phys. 77, $1173(2005)$.

${ }^{21}$ G. K. H. Madsen and D. J. Singh, Comp. Phys. Comm. 175, 67 (2006).

${ }^{22}$ G. Pizzi, D. Volja, B. Kozinsky, M. Fornari, and N. Marzari, Comp. Phys. Comm. 185, 422 (2014).

23 A. A. Mostofi, J. R. Yates, Y. S. Lee, I. Souza, D. Vanderbilt, and N. Marzari, Comp. Phys. Comm. 178, 685 (2008).

24 A. A. Mostofi, J. R. Yates, G. Pizzi, Y. S. Lee, I. Souza, D. Vanderbilt, and N. Marzari, Comp. Phys. Comm. 185, 2309 (2014).

25 G. Kresse and J. Furthmüller, Comp. Mat. Sci. 6, 15 (1996).

${ }^{26}$ G. Kresse and J. Furthmüller, Phys. Rev. B 54, 11169 (1996). 
${ }^{27}$ M. Gajdoš, K. Hummer, G. Kresse, J. Furthmüller, and F. Bechstedt, Phys. Rev. B 73, 045112 (2006).

${ }^{28}$ S. Poncé, E. R. Margine, C. Verdi, and F. Giustino, Comp. Phys. Comm. 209, 116 (2016).

29 P. Giannozzi, S. Baroni, N. Bonini, M. Calandra, R. Car, C. Cavazzoni, D. Ceresoli, G. L. Chiarotti, M. Cococcioni, I. Dabo, A. Dal Corso, S. de Gironcoli, S. Fabris, G. Fratesi, R. Gebauer, U. Gerstmann, C. Gougoussis, A. Kokalj, M. Lazzeri, L. Martin-Samos, N. Marzari, F. Mauri, R. Mazzarello, S. Paolini, A. Pasquarello, L. Paulatto, C. Sbraccia, S. Scandolo, G. Sclauzero, A. P. Seitsonen, A. Smogunov, P. Umari, and R. M. Wentzcovitch, J Phys: Cond. Matt. 21, 395502 (2009).

30 J. P. Perdew and A. Zunger, Phys. Rev. B 23, 5048 (1981).

31 J. P. Perdew, K. Burke, and M. Ernzerhof, Phys. Rev. Lett. 77, 3865 (1996).

32 A. I. Duff, T. Davey, D. Korbmacher, A. Glensk, B. Grabowski, J. Neugebauer, and M. W. Finnis, Phys. Rev. B 91, 214311 (2015).

33 G. Kresse and D. Joubert, Phys. Rev. B 59, 1758 (1999).

34 M. Methfessel and A. T. Paxton, Phys. Rev. B 40, 3616 (1989).

35 N. Marzari and D. Vanderbilt, Phys. Rev. B 56, 12847 (1997).

36 N. Marzari, A. A. Mostofi, J. R. Yates, I. Souza, and D. Vanderbilt, Rev. Mod. Phys. 84, 1419 (2012).

37 I. Souza, N. Marzari, and D. Vanderbilt, Phys. Rev. B 65, 035109 (2001).

38 D. M. Ceperley and B. J. Alder, Phys. Rev. Lett. 45, 566 (1980).

39 S. Baroni, P. Giannozzi, and A. Testa, Phys. Rev. Lett. 58, 1861 (1987).

40 S. Baroni, S. de Gironcoli, A. Dal Corso, and P. Giannozzi, Revi. Mod. Phys. 73, 515 (2001).

41 X. Gonze, Phys. Rev. B 55, 10337 (1997).

42 T. A. Mellan, A. I. Duff, and M. W. Finnis, Phys. Rev. B 98, 174116 (2018).

43 L. Paulatto, I. Errea, M. Calandra, and F. Mauri, Phys. Rev. B 91, 054304 (2015).

44 N. Bonini, M. Lazzeri, N. Marzari, and F. Mauri, Phys. Rev. Lett. 99, 176802 (2007).

45 X. Tang and B. Fultz, Phys. Rev. B 84, 054303 (2011).

46 R. Bauer, A. Schmid, P. Pavone, and D. Strauch, Phys. Rev. B 57, 11276 (1998).

47 Z. Lin, L. V. Zhigilei, and V. Celli, Phys. Rev. B 77, 075133 (2008).

${ }^{48}$ F. A. Modine, M. D. Foegelle, C. B. Finch, and C. Y. Allison, Phys. Rev B 40, 9558 (1989).

49 Y. Werman and E. Berg, Phys. Rev. B 93, 075109 (2016).

50 A. Auerbach and P. B. Allen, Phys. Rev. B 29, 2884 (1984).

51 Y. Werman, S. A. Kivelson, and E. Berg, Quant. Mat. 2, 1 (2017).

52 B. Sundqvist and V. K. Tolpygo, J Phys.: Conf. Ser. 500, 182040 (2014).

53 T. Sun and P. B. Allen, Phys. Rev. B 82, 224305 (2010).

${ }^{54}$ G. A. Slack, Sol. Stat. Phys. 34, 1 (1979).

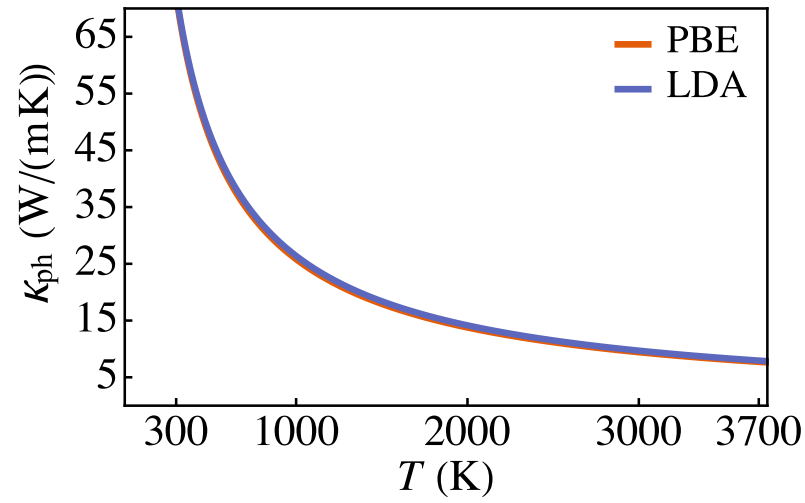

Figure 5. Comparison of the $\mathrm{ZrC}$ phonon thermal conductivity computed with the PBE and LDA exchange-correlation functionals at $a=4.667 \AA$.

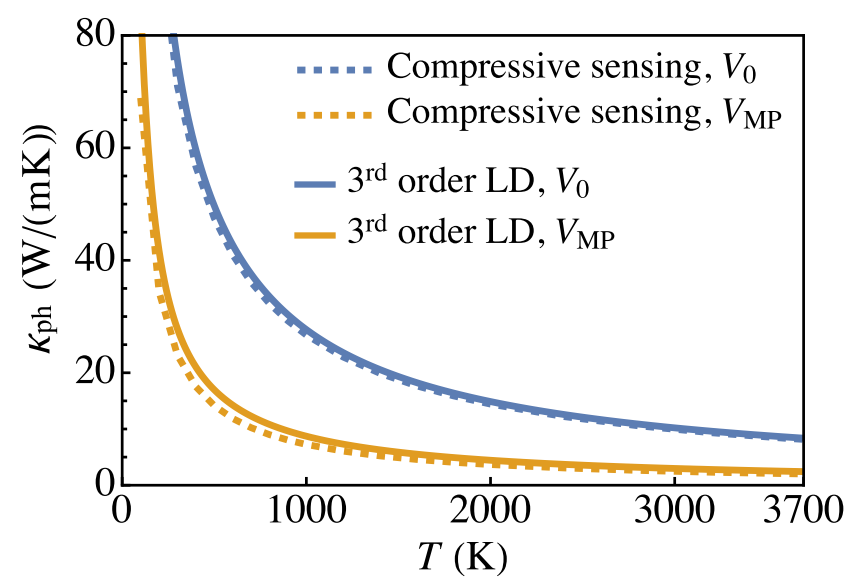

Figure 6. Comparison of conductivity from compressive sensing ${ }^{18}$ and third-order lattice dynamics (Phono3py ${ }^{12}$ ), at zero-temperature equilibrium and high-temperature volumes.

\section{APPENDIX}

\section{METHODOLOGICAL COMPARISONS}

The ZrC phonon conductivity calculated using the LDA and PBE exchange-correlation functionals are shown in Fig. 5. At fixed equal volumes, the difference in phonon conductivity is negligible.

In Fig. 6 the phonon thermal conductivities are compared from the compressive sensing ${ }^{18}$ and third-order ${ }^{12}$ lattice dynamics approaches. Compressive sensing predicts a similar but marginally smaller thermal conductivity.

The electrical conductivity from Wannier function, Bloch function and DFPT LDA DFT calculations is shown in Fig. 7. The conductivity with Wannier functions is expected to provide the most accurate predictions. 


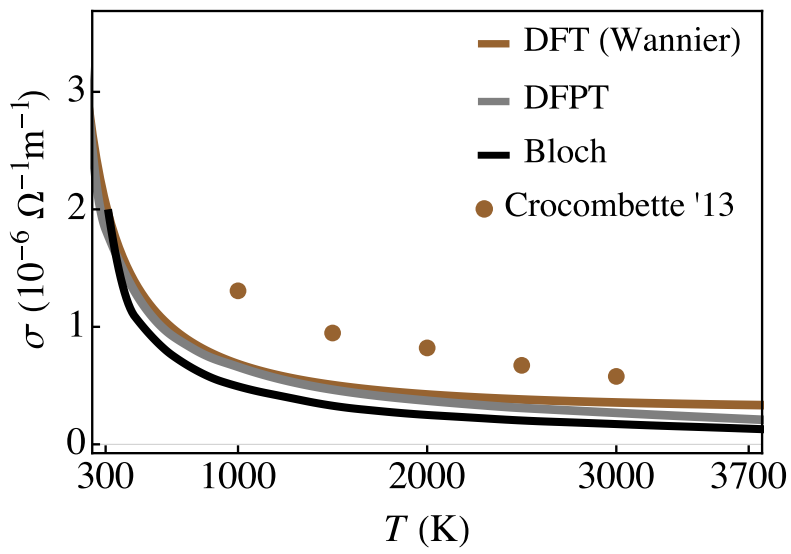

Figure 7. Comparison of computational approaches to determine electrical conductivity. 1) LDA + Wannier functions, 2) LDA + DFPT linear response, 3) LDA + Bloch functions, and 4) PBE + snapshots from classical potential $\mathrm{MD}^{11}$.

\section{PHONON SCATTERING GEOMETRIC CONSTRAINTS}

The effect of size constraints on phonon thermal conductivity is shown in Fig. 8. The geometry restriction effect is strongly temperature dependent, lowering the $\mathrm{ZrC}$ phonon conductivity most acutely at low temperature.

Temperature mean-free path saturation effects are expected to be weak until very high temperatures based on Fig. 9. At $300 \mathrm{~K}, \kappa_{\mathrm{ph}}$ from mean-free paths shorter than $10 \AA$ is negligible. At $1500 \mathrm{~K}$, less than $1 \%$ of the computed $\kappa_{\mathrm{ph}}$ arises from mean-free paths comparable to the lattice parameter. Even at $3800 \mathrm{~K}$, when the scattering rate is extremely high for the material, more than $90 \%$ of the computed $\kappa_{\mathrm{ph}}$ comes from mean-free paths greater than $a$, with approximately $0.2 \mathrm{Wm}^{-1} \mathrm{~K}^{-1}$ associated with mean-free paths shorter than the lattice parameter.

\section{PHONON RENORMALIZATION AND FOUR-PHONON SCATTERING}

At high temperature quasiharmonic and anharmonic frequency renormalization, along with four-phonon scattering, are expected to play increasingly more important roles. Quasiharmonic volume expansion generally lowers phonon frequencies, as shown in Fig. 10a-c, while anharmonic phonon renormalization tends to have the opposite effect and harden frequencies in $\mathrm{ZrC}$. Compared to three-phonon scattering, four-phonon scattering is a minor effect at low temperature $(300 \mathrm{~K})$, but becomes very prominent for temperatures exceeding $1500 \mathrm{~K}$ as shown in Fig. 10d-f. 


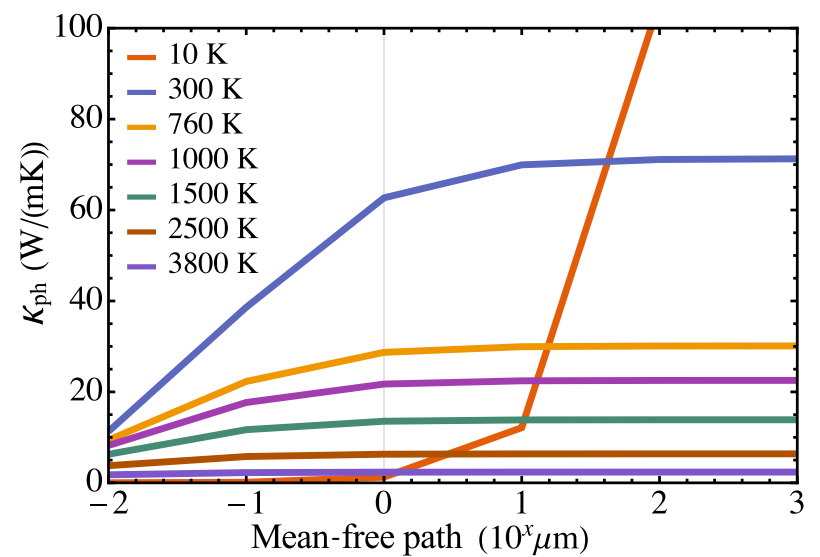

Figure 8. Grain-size effects estimated by geometric restrictions on phonon conductivity at a series of temperatures in perfect $\mathrm{ZrC}$. Phonon cutoff lengths span the interval $\left[10^{-2}, 10^{3}\right] \mu \mathrm{m}$.

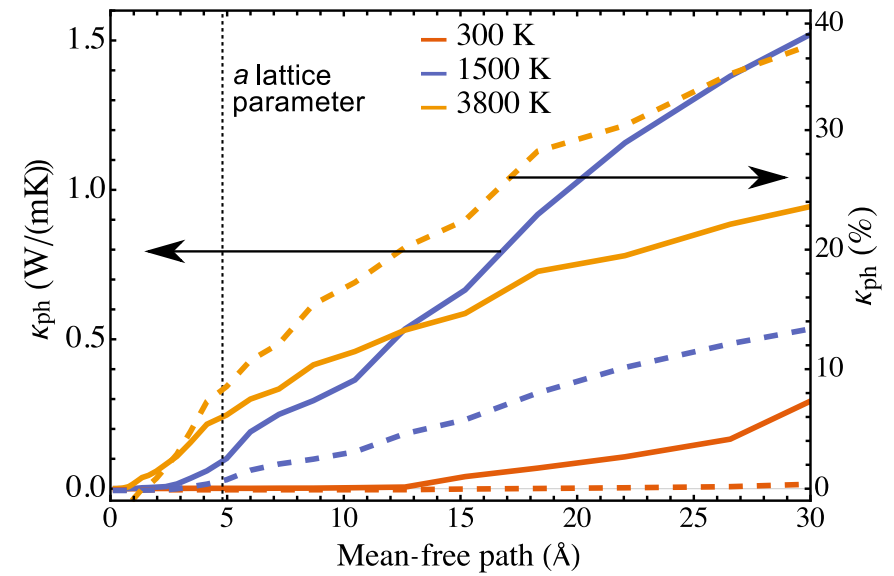

Figure 9. Accumulated phonon thermal conductivity as a function of mean-free path, shown in solid lines, left axis. Percentage accumulated phonon thermal conductivity is shown in dashed lines, right axis. 

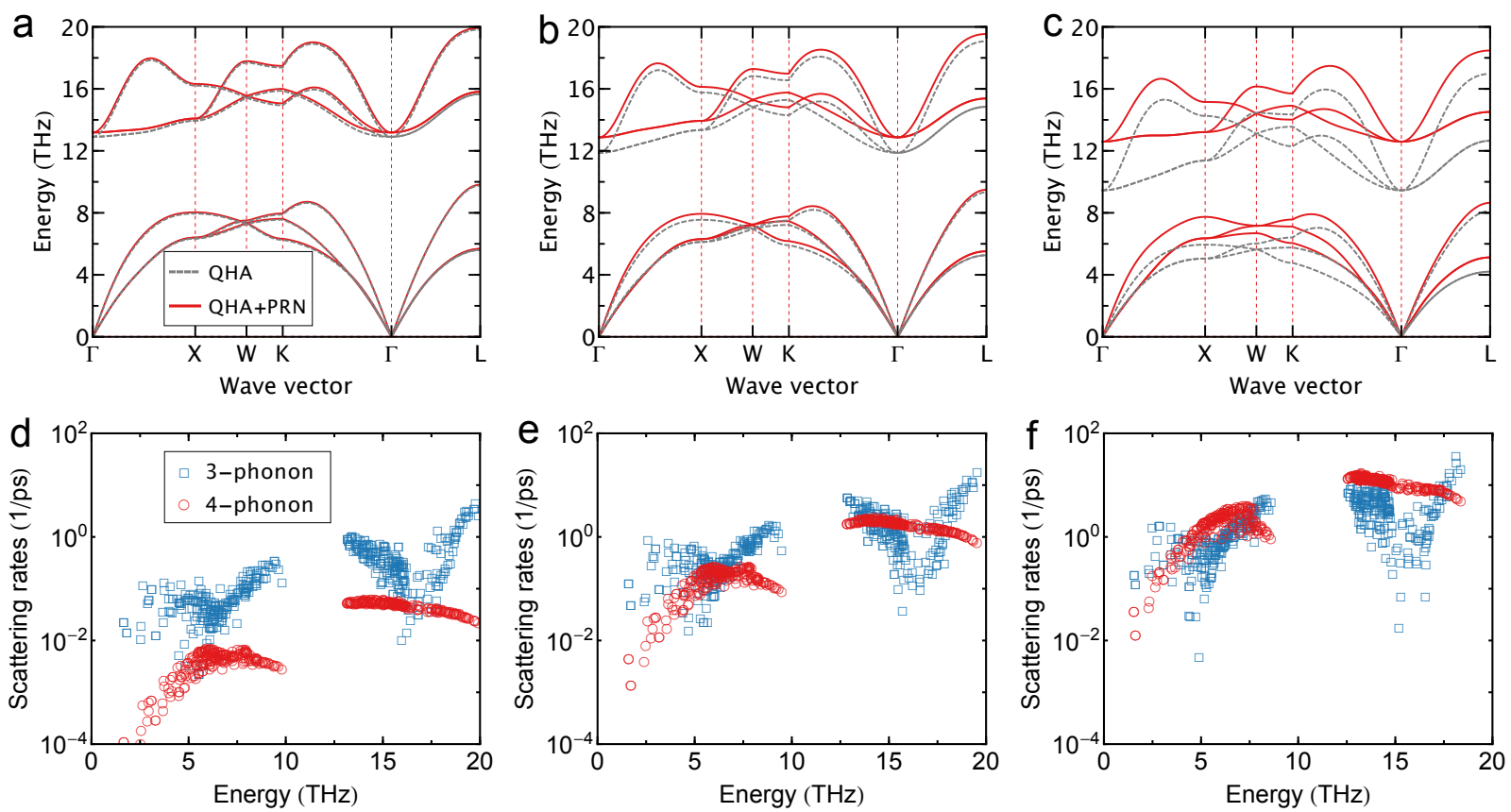

Figure 10. Phonon dispersion at $300 \mathrm{~K}$ (a), $1500 \mathrm{~K}$ (b) and $3800 \mathrm{~K}$ (c) at the quasiharmonic and quasiharmonic + renormalization levels of theory. Three and four-phonon scattering at $300 \mathrm{~K}(\mathrm{~d}), 1500 \mathrm{~K}(\mathrm{e})$ and $3800 \mathrm{~K}$ (f). 